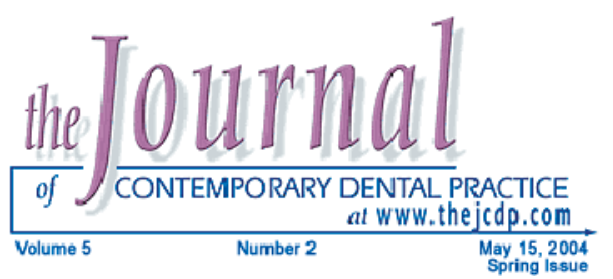

\title{
Storage Phosphor Plates: How Durable are they as a Digital Dental Radiographic System?
}

Aurelija Bedard; Tracy Dawn Davis; Christos Angelopoulos, DDS, MS

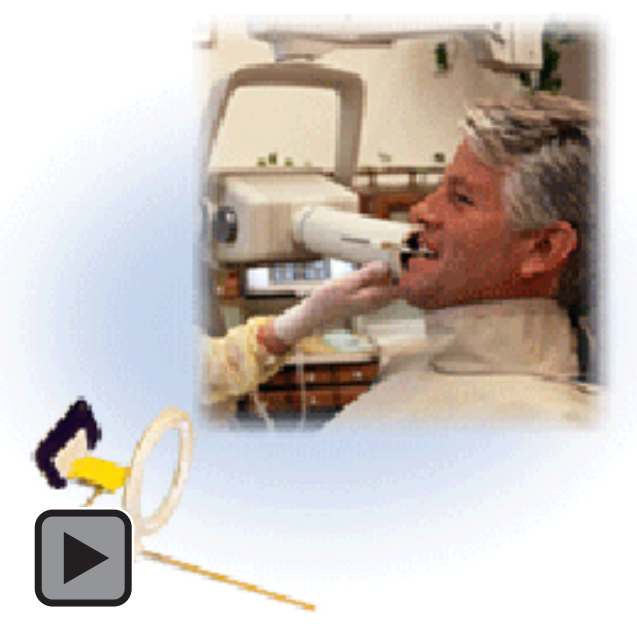

\section{Abstract}

The aim of this in vitro investigation was to evaluate the durability of storage phosphor plates (SPPs) as a digital dental imaging system and to detect the factors that may contribute to possible reduced durability. A total of 140 new SPPs were divided into groups and exposed to the effects of the various handling steps during the image acquisition. An additional group of plates joined the existing plates in the Radiology Clinic and tested the additive effect of all these factors plus the effect of positioning the plates in a patient's mouth. The images resulting from these SPPs were regularly evaluated for the appearance of scratches as a sign of wear and rated by an oral radiologist using a 6 point scale. Although the manufacturer claims the SPPs can be used indefinitely, we found $95 \%$ of our plates were rendered non-diagnostic after only 50 uses. The manufacturer's claim for indefinite use of the SPPs seems to be questionable.

Keywords: Digital radiography, photostimulable radiography, storage phosphor plates, durability

Citation: Bedard A, Davis TD, Angelopoulos C. Storage Phosphor Plates: How Durable are they as a Digital Dental Radiographic System? J Contemp Dent Pract 2004 May;(5)2:057-069.

(C) Seer Publishing 


\section{Introduction}

Accurate oral diagnosis and treatment are very closely linked to the quality of dental radiographs. ${ }^{1}$ Consequently, utilization of radiographic images of reduced quality may hide or obscure crucial information which, in turn, may have an effect on the diagnosis and possibly treatment planning. On the other hand, if useful diagnostic details are not completely obscured in a radiographic image of reduced quality, this may lead to a "remake" of the radiograph resulting in unnecessary radiation exposure to the patient.

Digital radiography has been introduced into dental practice as the alternative to film-based radiography since it can reduce radiation exposure to the patient without compromising image quality. In fact, digital radiography has been shown to be as accurate as conventional film-based radiography in diagnosing caries periapical lesions and periodontal disease..$^{2-9}$

Digital radiography utilizes the same projection technology as film-based radiography; however, the images are recorded by an electronic sensor. There are two main categories of intraoral sensors: direct sensor systems and storage phosphor plates (SPPs).

The direct sensor systems or charge-coupleddevice (CCD)-based systems include a CCD sensor, a processing unit, a digital interface card, a computer, and software. The CCD sensors

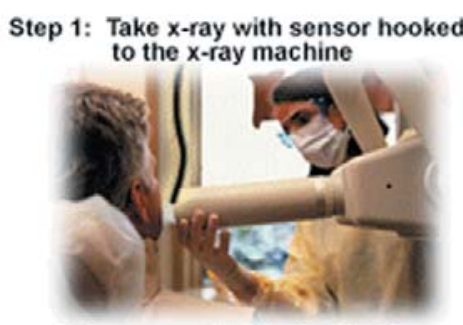

Charge-coupled-devise System

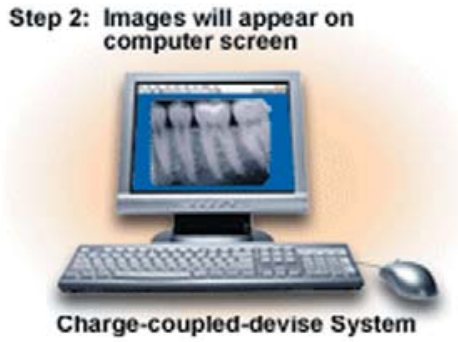

are $\mathrm{x}$-ray-sensitive or light-sensitive pixels arranged in lines on a rectangular base. The more pixels, the higher the quality of the captured image. Sensors that include $x$-ray sensitive cells or pixels can generate a voltage in proportion to the amount of $x$-ray photons striking them. The CCD charge is collected in a serial fashion from pixel to pixel to the readout amplifier. The result is a two-dimensional array of gray shades that can be displayed on a monitor. ${ }^{10}$ Sensors that include light-sensitive pixels convert the $x$-ray energy to light with the integration of a scintillation screen in front of the CCD cells. In this case, the CCD charge is proportional to the light emitted from the screen which, in turn, is proportional to the energy of the x-ray photons. The drawback of the lightsensitive CCD sensor is it is generally thicker than the $\mathrm{x}$-ray sensitive sensor.

SPPs include a reusable photostimulated screen, a readout scanner, a photomultiplier tube, a digital interface card, a computer, and software. When $\mathrm{x}$-ray photons strike the phosphor layer included on the plate, a latent image is formed. The phosphor screen releases the stored energy as light photons when scanned by the readout device by means of photostimulation. The intensity of the light emitted is proportional to the $x$-rays absorbed by the plate. The photomultiplier tube is used to convert small amounts of light into an amplified electric signal. ${ }^{10}$ The resulting signal is digitized by an analog-todigital converter (ADC), which determines the number of shades of gray in the image.

The main advantages of both CCD sensors and SPPs include reduction in radiation dose to the patient and availability of a variety of image enhancements. CCD sensors offer more rapid image acquisition. Cost of the equipment, stiffness of the sensor, and presence of a cord attachment between the sensor and computer are

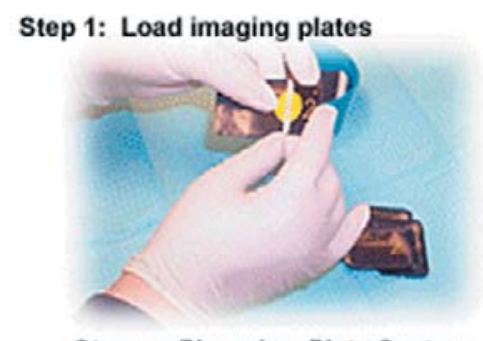

Storage Phosphor Plate System

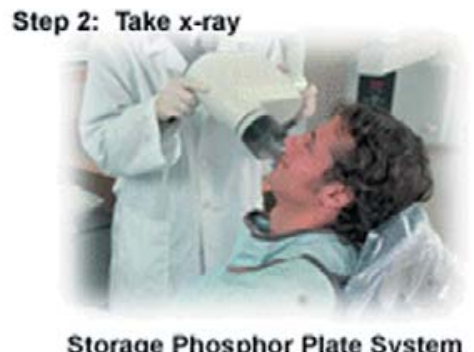

Step 3: Mount plates in carousel

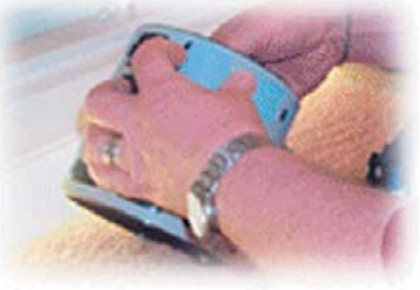

Storage Phosphor Plate System

Step 4: Place in scanner \& scan images

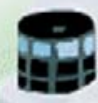

Storage Phosphor Plate System 
Step 6: Erase imaging plates for reuse

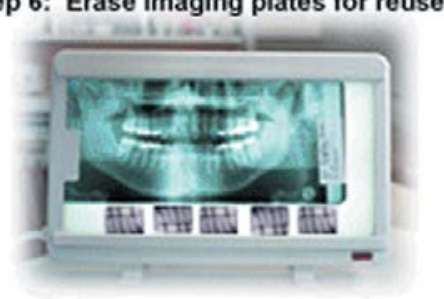

Storage Phosphor Plate System

Step 5: Images appear on computer

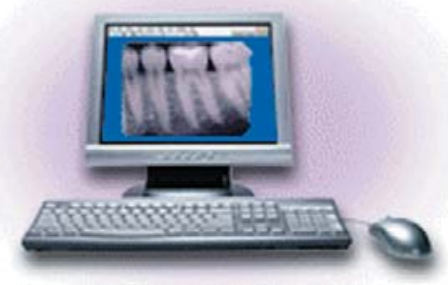

Storage Phosphor Plate System the main drawbacks of the CCD sensors. SPPs demonstrate wider exposure latitude than do CCD sensors. Flexibility of these plates and absence of an electrical cord are other advantages. The primary disadvantage of SPPs is the need for additional time to readout the image. ${ }^{11}$ The need for a readout device to scan the plates adds to the cost of the system. Also, plates must be exposed to light to erase the residual image before reusing them. Lastly, the resolution of storage phosphor images is reduced compared to CCD images since their pixel size is slightly larger. ${ }^{11}$ Despite advancements in digital radiography, the resolution of digital images in general is still lower than radiographic film. ${ }^{12}$

The above mentioned similarities of SPPs to radiographic film (no cords attached, flexibility, thickness) were taken into account in order to secure a smooth transition from film-based radiography to digital radiography when the decision to adopt the Denoptix storage phosphor system (Dentsply International, Des Plaines, IL 60018) as the new imaging system at the University of Missouri-Kansas City, School of Dentistry was made. Since the integration of such a system (August 2001), dental imaging is now completely digital in this facility. As our experience with SPPs grew over time, issues that were not considered earlier came into surface, i.e., the digital

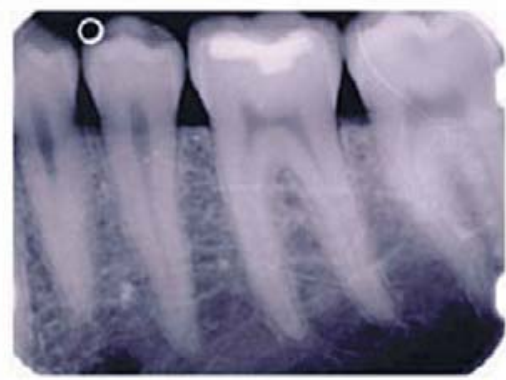

Figure 1. Digital periapical radiographic image showing several scratches varying in length, width, and intensity. images from SPPs which had been repeatedly used seemed to demonstrate a lower quality due to the appearance of scratches. These scratches can some times render the resulting image completely non diagnostic and may lead to a remake of the radiograph (Figure 1).

Our experience with SPPs indicated the durability of this imaging system may be limited despite the manufacturer's claim the SPPs can be used indefinitely.

A search of the literature yielded no studies addressing the durability of digital systems in general, nor that of SPPs. If indeed there is a durability issue, the practitioners as well as the manufacturer should be aware of it.

The aim of this in vitro investigation was to evaluate the durability of SPPs as a digital dental imaging system and to detect the factors that may contribute to possible reduced durability.

\section{Materials and Methods}

The utilization of SPPs in a radiographic system requires several steps in which the plates are handled prior to image acquisition making them vulnerable to scratches.

The SPPs are first placed into protective plastic barriers, then in the film holder of the positioning devices, next in the patient's mouth, and lastly, mounted on the drum of the Denoptix System before scanning with the scanner. During the above mentioned steps, the SPPs may suffer scratches by either the fingernails of the various workers involved or the patient's teeth or both.

In order to detect the possible contribution of each one of the above steps on the appearance of scratches on SPPs, four groups of SPPs were introduced, each of which corresponded to a handling step:

- Group 1: (20 plates, \#2 in size) (Figure 2) These plates were placed repeatedly in plastic barriers by the same worker who is responsible for this duty in the Radiology Clinic, under the same conditions applied to the clinic's SPPs.

- Group 2: (20 plates, \#2 in size) (Figure 3). These plates were placed repeatedly in XCP 


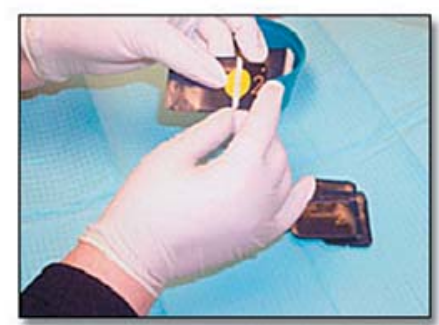

Figure 2. Placement of the SPPs in protective barriers.

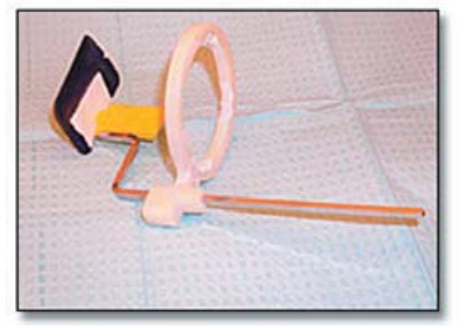

Figure 3. A SPP in the XCP film holding device.

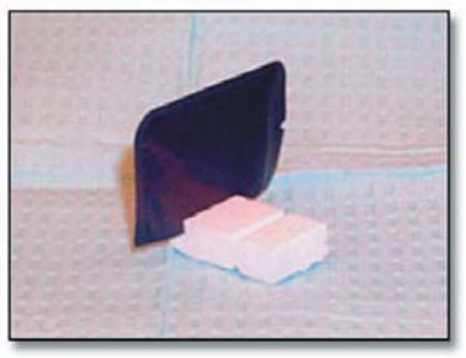

Figure 4. A SPP placed in a styrofoam film holder.

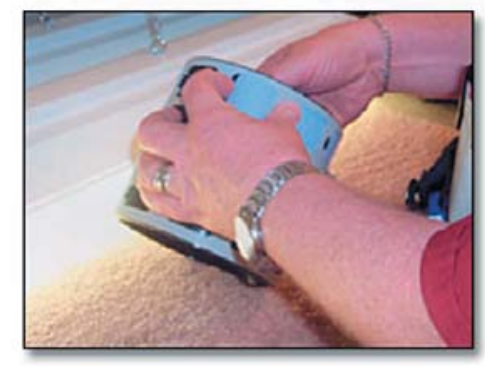

Figure 5. SPPs during mounting on the scanning drums of the Denoptix system.

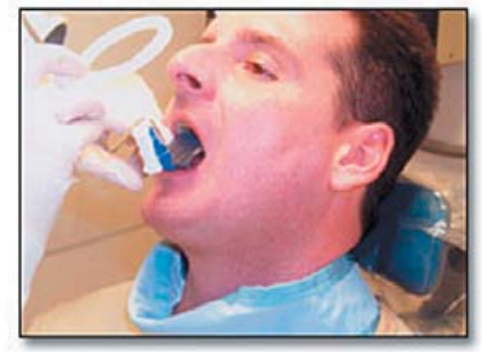

Figure 6. SPP during positioning. film holders by one dental student, exactly the same way that he/she would have carried out the above task with a patient.

- Group 3: (20 plates, \#2 in size) (Figure 4). The SPPs of this group were repeatedly placed by one dental student (different person) into disposable (styrofoam) film holder devices, exactly the same way that he/she would have carried out the above task with a patient.

- Group 4: (20 plates, \#2 in size) (Figure 5). These plates were repeatedly mounted onto the drums of the Denoptix System by the same operator who carries out the above duty at the Radiology Clinic under the same conditions that he/she applies in the clinic.

Lastly, in order to detect the possible additive effect of these factors and the effect of the actual positioning of the SPPs in the patient's mouth, a fifth group was introduced, composed by 60 (\#2 in size) plates which were marked for identification and added to the existing, circulating SPPs being used in the Radiology Clinic. These SPPs were handled exactly the same way as the rest of the clinic's plates (Figure 6). All of the SPPs used in this experiment were new.

A custom software program at the UMKC School of Dentistry is used to monitor the number of exposures per plate, which in turn represented the number of repetitions for each of Groups 1-4. For example, if there were 12 exposures per plate during the first two weeks of the experiment, this would have indicated each plate had been handled 12 times for barrier application, had been placed in a film holder device 12 times, and had been scanned 12 times. Therefore, each of the plates of the groups 1-4 would have been exposed 12 times to the corresponding handling steps of each group.

The SSPs included in the experiment were evaluated every 15 days for the duration of the experiment for signs of wear (appearance of scratches). To do so, the primary investigator exposed the SPPs (Groups 1-5) to radiation under standardized conditions ( $70 \mathrm{kV}, 15 \mathrm{~mA}, 21 \mathrm{imp}$ and fixed focal spot to plate distance) using the same $x$-ray unit (Gendex 2000). The resulting digital images were stored in groups of 20 in five different loca- 
tions for the five different experimental groups. Although the overall number of images that were finally produced and available for assessment was supposed to be 840 [140 images for the groups 1-5X 6 times of image production for evaluation (once every 15 days)], this number was in fact 821. Seven SPPs were lost from Group 5 at various stages of the experiment.

When the experiment was terminated at the end of 10 weeks, each SSP in the experimental groups was used 50 times.

\section{Image Evaluation}

An oral and maxillofacial radiologist evaluated the 821 digital images that resulted from the exposed SSPs of all groups (1-5) in 8 different sessions with weekly intervals ( 7 sessions of 100 images, 1 session of 121 images). The digital images were assessed for the presence of scratches and diagnostic quality on a 15" LCD monitor (Gateway 2000, North Sioux City, SD) under optimal viewing conditions. A 6 point scale (0-5) was used for the evaluation: $0=$ no scratches present/fully diagnostic image and $5=$ several scratches present/completely non diagnostic image. Oral and written instructions were provided (Table 1).
Also, examples of plates demonstrating variable degrees of wear including all classifications of the 6 point scale were available for the rater as a quick reference (Figure 7 ). The images were brought on screen in groups of 20 and the rater was permitted to magnify each individual image to full screen and to enhance it for brightness/contrast and/or gamma during the evaluation session.

Before each rating session, the rater was calibrated for the evaluation process using a small number of randomly selected images.

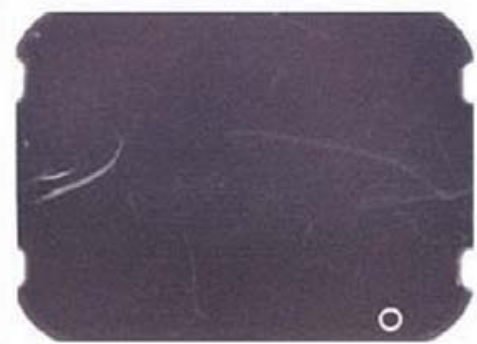

Figure 7. An example of a SSP rated as 5 due to the appearance of several scratches that would most likely result in a non-diagnostic image.
The order of the

series of images that were evaluated in each session was randomized, and the examiner was blind for the experimental group of origin of the digital images as well as the time frame the images were obtained.

Table 1. Guidelines for rating storage phosphor plates (SPPs).

0 No scratches at all, the image is totally diagnostic.

1 Minimal wear.

Less than 4 short faint scratches present, or less than 2 scratches present including one longer.

$25-8$ short faint scratches present, or less than 3 including 1-2 longer, or less than 3 including 1-2 brighter.

3 5-8 short scratches including 2-3 brighter ones, or 3-6 including 1-2 brighter and longer, or less than 3 longer, brighter and wider scratches.

4 Plate is almost non-diagnostic with more than 8 short scratches, or 5-8 including up to 3 longer, and/or brighter ones.

5 Plate is non-diagnostic.

Several scratches present varying in length, width and brightness, and cover most of the plate's surface.

Note: Table one contains written instructions for the rater (an oral and maxillofacial radiologist) for the evaluation of the SPPs. 


\section{Statistical Analysis}

Descriptive statistics were used to assess the effect of time and repeated use on the durability of the SPPs. Descriptive statistics were also used to evaluate the contribution of each individual step of handling of the SSPs on the appearance of scratches that compromise the diagnostic efficacy of the SPPs.

\section{Percentage of Undiagnostic SPPs (rated 4 or 5 )}
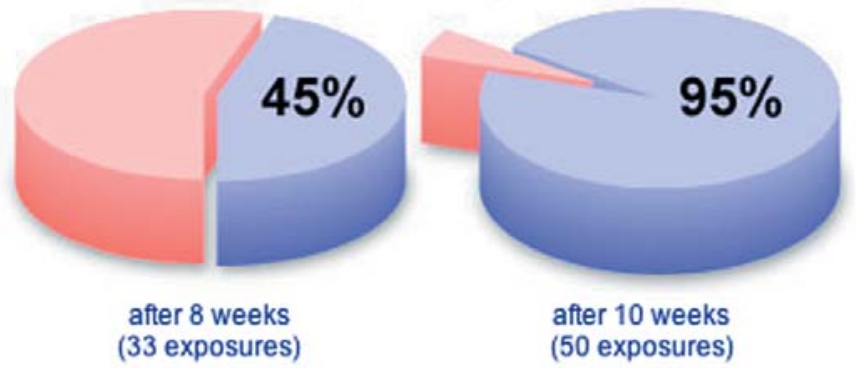

Figure 8. Pie graph showing the percentage of SPPS that were rated 4 or 5 after 33 exposures and after 50 exposures, respectively.

\section{Results}

Figure 8 illustrates averaged data from Group 5 (SPPs that were being used as the remaining plates of the Radiology Clinic). Yellow demonstrates percentages of the SPPs that have been rated with the highest scores (4 or 5 ) and considered virtually non-diagnostic. The percentage of the non-diagnostic SPPs doubled from 33 exposures (uses) to 50 exposures.

The comparative average rating of the SPPs of all groups over the duration of the experiment is shown in Figure 9. Groups 1, 2, 3 (barrier placement, XCP film holder, and disposable film holder device) contribute minimally to the wear of the SPPs. Their average rating after 50 exposures was below 1. In contrast placing the SPPs on the drums for scanning contributes considerably to the appearance of scratches. The additive effect of all the factors (Group 5) is demonstrated with yellow.

\section{Discussion}

This in vitro investigation addressed two closely related questions:
(1) Is there a durability issue for the SPPs that could potentially result in digital images of compromised or reduced quality?

(2) If such an issue exists, which are the contributing factors during the SPPs' handling process?

The significance of the first question lies in the fact that a (1) digital radiographic image compromised in quality could potentially lead to a compromised treatment approach if relevant diagnostic information is completely obscured or (2) a remake of the radiograph will result in unnecessary exposure to the patient. The compromised quality in the digital images made with SPPs will be in the form of scratches (Figure 1) varying in length, width, location, and intensity. Although these scratches will not always interfere with diagnostic information in a radiograph, sometimes they will. In our study, Group 5 was the one to address the first question.

\section{Average Rating of SPP Quality Over Time}

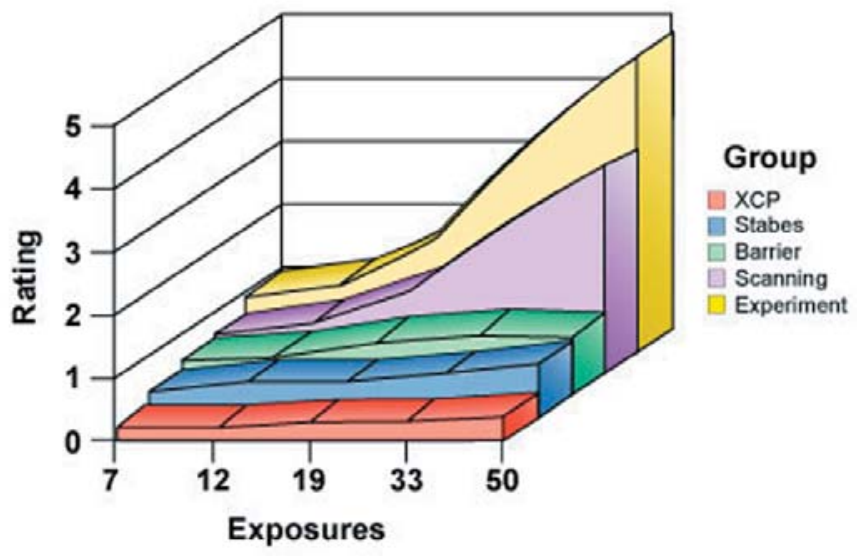

Figure 9. Average rating of the SPPs of Groups 1-5 for the duration of the experiment.

The graph in Figure 9 clearly shows that as the number of uses per plate increases, so does the appearance of scratches on the resulting digital images. The digital images produced after several uses of the SPPs scored higher on the 6 point scale and tend to become less diagnostic. In fact, a striking $95 \%$ of the SPPs in Group 5 were rated either 4 or 5 by the rater which means the appearance of scratches on these images was such that rendered them non-diagnostic after 50 uses (Figure 8). If this is the case, the SPPs 
must be checked for wear frequently and replaced often. It is worthy of mentioning that during busy times in our school this number can be reached in less than six weeks. Moreover, there is a cost issue if indeed most of these plates need to be retired after 50 uses.

Experimental Groups 1-4 contributed in investigating the factors during the handling of the SPPs that had an effect in the appearance of scratches. It appears all handling steps contributed to the wear of the SPPs (Figure 9), and this effect is more than likely additive, with the handling during scanning of the plates probably contributing the most. As mentioned above, the SPPs are thin (thinner than film) and trying to get a grip in a semi-dark environment as the scanning room is somewhat challenging. Moreover, after scanning, the plates are firmly attached on the scanning drum and may become scratched while attempting to disengage them.

The following three factors could have influenced our study results:

1. Inexperienced operators

2. Inexperienced staff

3. The utilization of SPPs as a radiographic system

4. SPPs vulnerability to wear
A department of radiology in a dental school is an educational facility so the operators of the SPPs are mostly dental students with different levels of experience. As a result, the wear of the SPPs may be expedited because of the inexperienced users of the system. A similar study in which the operators will be experienced dental personnel from private practice will be used in order to investigate the possible effect of inexperience on the SPP wear. Also, the Radiology Department personnel are involved in various steps of the SPPs handling as well. Although they were trained on the utilization of the system, regular reinforcement of the need for careful handling may be necessary. Lastly, the SPPs may be too sensitive to be used in a multi-step imaging process and in such a small space as the mouth. There is room for improvement in SPPs as the manufacturer needs to consider the durability issue as well as enhance the physical characteristics of the plates.

Unfortunately, our literature search yielded no other studies addressing this issue so our results cannot be compared. However, this issue requires further investigation.

\section{Conclusion}

This study suggests the manufacturer's claim for indefinite use of the SPPs as a digital imaging system is questionable. It seems our effort at the University of Missouri-Kansas City, School of Dentistry should focus on taking better care of the SPPs, especially handling during scanning, although a better solution may be the manufacture of an improved SPP.

\section{References}

1. Goaz and White. Oral radiology principles and interpretation. 3rd ed. St. Louis: Mosby, 1994.

2. Wenzel A. Digital radiography and caries diagnosis. Dentomaxillofac Radiol. 1998 Jan;27(1):3-11. Review.

3. Tyndall DA, Ludlow JB, Platin E, et. al. A comparison of Kodak Ektaspeed Plus film and the Siemens Sidexis digital imaging system for caries detection using receiver operating characteristic analysis. Oral Surg Oral Med Oral Pathol Oral Radiol Endod. 1998 Jan;85(1):113-8.

4. Naitoh M, Yuasa $\mathrm{H}$, Toyama M, et. al. Observer agreement in the detection of proximal caries with direct digital intraoral radiography. Oral Surg Oral Med Oral Pathol Oral Radiol Endod. 1998 Jan;85(1):107-12.

5. Price C, Ergul N. A comparison of a film-based and a direct digital dental radiographic system using a proximal caries model. Dentomaxillofac Radiol. 1997 Jan;26(1):45-52.

6. Kullendorff $\mathrm{B}$, Nilsson M, Rohlin M. Diagnostic accuracy of direct digital dental radiography for the detection of periapical bone lesions: overall comparison between conventional and direct digital radiography. Oral Surg Oral Med Oral Pathol Oral Radiol Endod. 1996 Sep;82(3):344-50. 
7. Kullendorff B, Nilsson M. Diagnostic accuracy of direct digital dental radiography for the detection of periapical bone lesions. II. Effects on diagnostic accuracy after application of image processing. Oral Surg Oral Med Oral Pathol Oral Radiol Endod. 1996 Nov;82(5):585-9.

8. Wenzel A, Borg E, Hintze $\mathrm{H}$, et al. Accuracy of caries diagnosis in digital images from chargecoupled device and storage phosphor systems: an in vitro study. Dentomaxillofac Radiol. 1995 Nov;24(4):250-4.

9. Furkart AJ, Dove SB, McDavid WD, et al. Direct digital radiography for the detection of periodontal bone lesions. Oral Surg Oral Med Oral Pathol. 1992 Nov;74(5):652-60.

10. Sanderink GC, Miles DA. Intraoral detectors. CCD, CMOS, TFT, and other devices. Dent Clin North Am. 2000 Apr;44(2):249-55, v.

11. Versteeg $\mathrm{CH}$, Sanderink GC, van der Stelt PF. Efficacy of digital intra-oral radiography in clinical dentistry. J Dent. 1997 May-Jul;25(3-4):215-24. Review.

12. Wenzel A, Grondahl HG. Direct digital radiography in the dental office. Int Dent J. 1995 Feb;45(1):2734. Review. Erratum in: Int Dent J 1995 Dec;45(6):391.

\section{About the Authors}

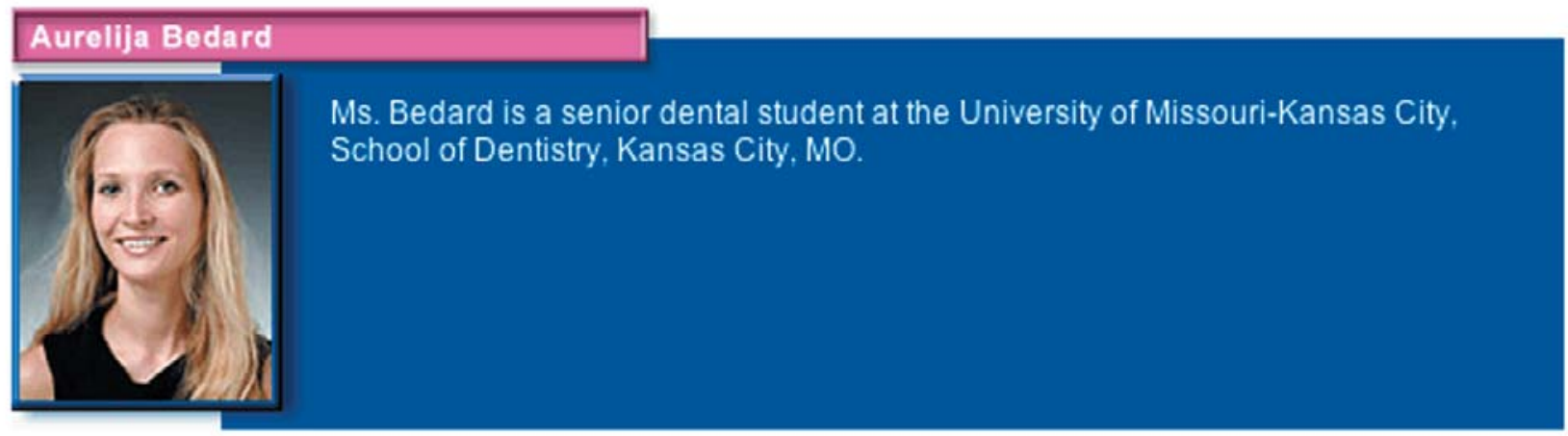

\section{Tracy Dawn Davis}

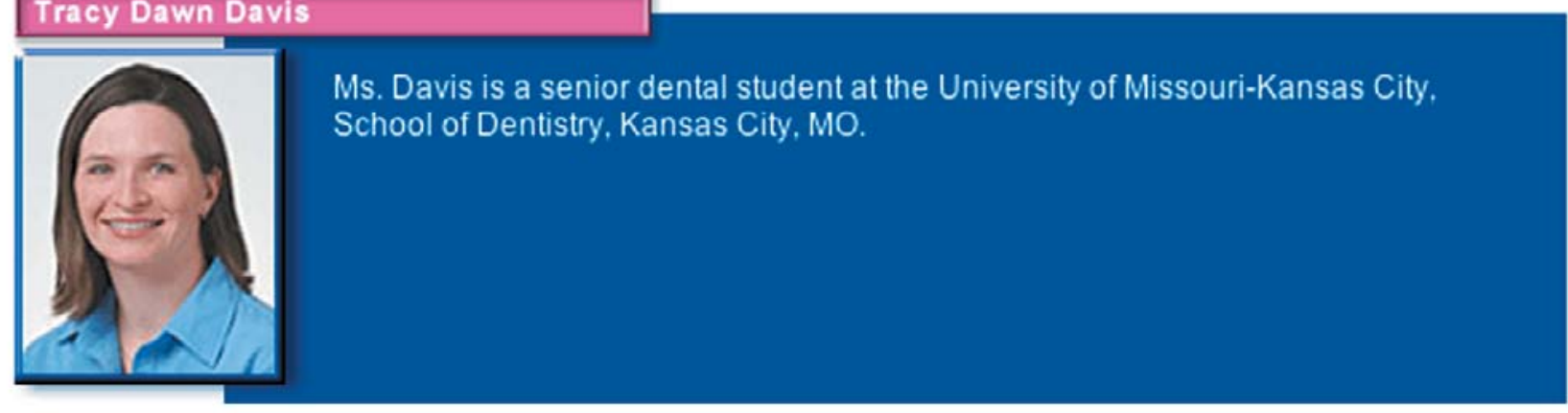

\section{Christos Angelopoulos DDS, MS}

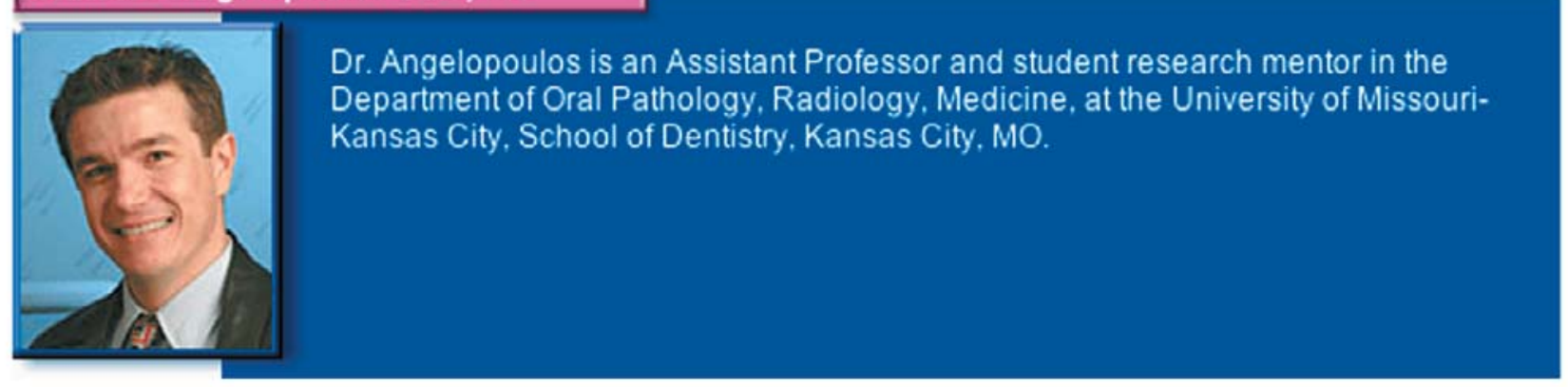

